\title{
A correlation coefficient for complex mass function in evidence theory
}

\author{
Fuyuan Xiao, Member, IEEE
}

\begin{abstract}
In this paper, a complex evidential correlation coefficient is proposed for complex mass functions. The complex conflict coefficient satisfies the desirable properties for conflict measurement, including the non-negativity, symmetry, boundedness, extreme consistency, and insensitivity to refinement. A numerical example is provided to illustrate the proposed method.
\end{abstract}

Index Terms-Generalized Dempster-Shafer evidence theory, Complex evidential correlation coefficient, Complex conflict coefficient, Complex mass function, Complex basic belief assignments.

\section{INTRODUCTION}

A recent work in terms of the generalization of DempsterShafer evidence (GDSE) theory is presented where a new concept of complex belief function is defined based on the complex numbers [1]. The GDSE theory is capable of giving expression to the data fluctuations at a given time phase in the course of execution. Moreover, it has the ability to handle uncertainty and imprecision when the data occur concurrently accompanied by variations against to data's phase or periodicity. In particular, when the complex basic belief assignments are degenerated from complex numbers to real numbers, the GDSE theory will degrade into the DSE theory with the condition that the conflict coefficient is less than one. Therefore, the GDSE theory can provide a more promising framework to model and cope with uncertain information.

In evidence theory, because the conflict measure plays an important role to manage the conflict, a lot of methods were presented in the past few years. In this paper, inspired by Jiang's correlation coefficient [2], a complex evidential correlation coefficient (CECC) is proposed which can measure the correlation coefficient between CBBAs in GDSE theory. The new CECC is a generalization of the traditional correlation coefficient. In particular, when the CBBAs are degenerated from complex numbers to real numbers, the CECC degrades into the Jiang's correlation coefficient. Hence, the CECC has more capability to measure the correlation between evidences. In the context of CECC, a complex conflict coefficient is proposed to measure the conflict degree between CBBAs. We then discuss that the complex conflict coefficient has the desirable properties for conflict measurement, including the nonnegativity, symmetry, boundedness, extreme consistency, and insensitivity to refinement. A numerical example is provided to illustrate the proposed method.

Corresponding author: Fuyuan Xiao.

F. Xiao is an Associate Professor at the School of Computer and Information Science, Southwest University, No.2 Tiansheng Road, BeiBei District, Chongqing, 400715, China (e-mail: xiaofuyuan@swu.edu.cn).
The rest of this paper is organized as follows. The complex mass function is introduced in Section II. The complex evidential correlation coefficient and conflict coefficient between CBBAs are proposed in Section III, in which their properties are discussed. Section IV provides the numerical example to illustrate the proposed method.

\section{COMPLEX MASS FUNCTION}

A generalization of Dempster-Shafer evidence (GDSE) theory is presented recently, in which a new concept of complex belief function is defined based on the complex numbers [1].

Let $\Omega$ be a set of mutually exclusive and collective nonempty events, defined by

$$
\Omega=\left\{e_{1}, e_{2}, \ldots, e_{i}, \ldots, e_{n}\right\},
$$

where $\Omega$ represents a frame of discernment.

The power set of $\Omega$ is denoted by $2^{\Omega}$, in which

$$
\begin{array}{r}
2^{\Omega}=\left\{\emptyset,\left\{e_{1}\right\},\left\{e_{2}\right\}, \ldots,\left\{e_{n}\right\},\left\{e_{1}, e_{2}\right\}, \ldots,\left\{e_{1},\right.\right. \\
\left.\left.e_{2}, \ldots, e_{i}\right\}, \ldots, \Omega\right\},
\end{array}
$$

and $\emptyset$ is an empty set.

\section{Definition 1 (Complex mass function)}

A complex mass function $\mathrm{IM}$ in the frame of discernment $\Omega$ is modeled as a complex number, which is represented as a mapping from $2^{\Omega}$ to $\mathbb{C}$, defined by

$$
\mathrm{M}: \quad 2^{\Omega} \rightarrow \mathbb{C},
$$

satisfying the following conditions,

$$
\begin{aligned}
\mathrm{M}(\emptyset) & =0, \\
\mathbb{M}(A) & =\mathbf{m}(A) e^{i \theta(A)}, \quad A \in 2^{\Omega} \\
\sum_{A \in 2^{\Omega}} \mathrm{M}(A) & =1,
\end{aligned}
$$

where $i=\sqrt{-1} ; \mathbf{m}(A) \in[0,1]$ representing the magnitude of the complex mass function $\mathbb{M}(A) ; \theta(A) \in\left[-\frac{\pi}{2}, \frac{\pi}{2}\right]$ denoting a phase term.

In Eq. (4), $\mathrm{M}(A)$ can also be expressed in the "rectangular" form or "Cartesian" form, denoted by

$$
\operatorname{MM}(A)=x+y i, \quad A \in 2^{\Omega}
$$

with

$$
\sqrt{x^{2}+y^{2}} \in[0,1] .
$$

By using the Euler's relation, the magnitude and phase of the complex mass function $\mathrm{M}(A)$ can be expressed as

$$
\mathbf{m}(A)=\sqrt{x^{2}+y^{2}} \text {, and } \theta(A)=\arctan \left(\frac{y}{x}\right),
$$




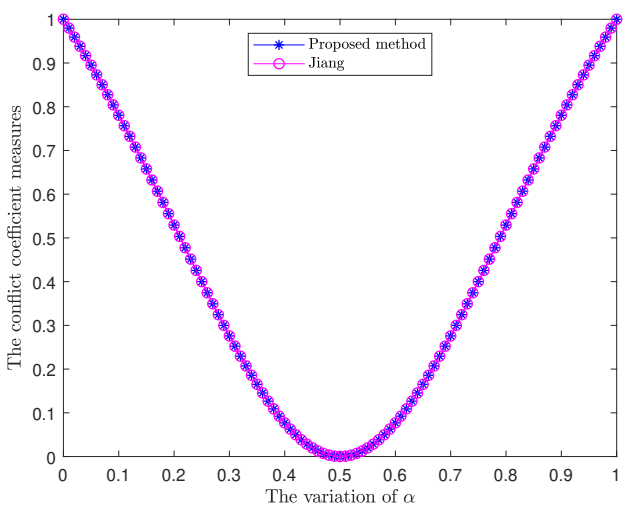

(a) $\omega=\left\{\mathcal{A}_{2}\right\}$.

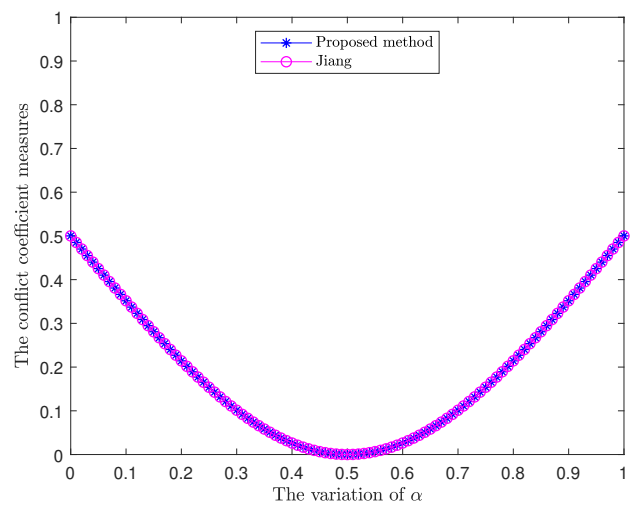

(b) $\omega=\left\{\mathcal{A}_{1}, \mathcal{A}_{2}\right\}$.

Fig. 1. The comparison of different correlation coefficient measures in Example 1.

where $x=\mathbf{m}(A) \cos (\theta(A))$ and $y=\mathbf{m}(A) \sin (\theta(A))$.

The square of the absolute value for $\mathrm{M}(A)$ is defined by

$$
|\mathrm{M}(A)|^{2}=\mathrm{M}(A) \overline{\mathrm{M}}(A)=x^{2}+y^{2},
$$

where $\overline{\mathrm{M}}(A)$ is the complex conjugate of $\mathrm{M}(A)$, such that $\overline{\mathrm{M}}(A)=x-y i$.

These relationships can be then obtained as

$$
\mathbf{m}(A)=|\mathrm{M}(A)|, \text { and } \theta(A)=\angle \mathrm{M}(A),
$$

where if $\operatorname{M}(A)$ is a real number (i.e., $y=0$ ), then $\mathbf{m}(A)=$ $|x|$.

If $|\operatorname{M}(A)|\left(A \in 2^{\Omega}\right)$ is greater than zero, $A$ is called a focal element of the complex mass function. The value of $|\mathbb{M}(A)|$ represents how strongly the evidence supports $A$.

The complex mass function $\mathrm{IM}$ modeled as a complex number in the generalized Dempster-Shafer (GDS) evidence theory can also be called a complex basic belief assignment (CBBA). When $\operatorname{M}(A)$ degrades into a real number, a CBBA will degrades into a BBA.

\section{A COMPLEX EVIDENTIAL CORRELATION COEFFICIENT}

Definition 2 (Complex evidential correlation coefficient between $C B B A s$ )

The complex evidential correlation coefficient (CECC) between $C B B A$ s $\mathbb{M}_{1}$ and $\mathbb{M}_{2}$, denoted as $\mathbb{C}\left(\overrightarrow{\mathrm{M}}_{1}, \overrightarrow{\mathrm{M}}_{2}\right)$ is defined by

$$
\mathbb{C}\left(\overrightarrow{\mathrm{M}}_{1}, \overrightarrow{\mathrm{M}}_{2}\right)=\frac{\sqrt{\left\langle\overrightarrow{\mathrm{M}}_{1}, \overrightarrow{\mathrm{M}}_{2}\right\rangle\left\langle\overrightarrow{\mathrm{M}}_{2}, \overrightarrow{\mathrm{M}}_{1}\right\rangle}}{\left\|\overrightarrow{\mathrm{M}}_{1}\right\|\left\|\overrightarrow{\mathrm{M}}_{2}\right\|}
$$

The CECC is a generalization of Jiang's correlation coefficient [2].

Theorem 1 The CECC has the properties of the nonnegativity, non-degeneracy, symmetry and boundedness.

Property 1 Let $\mathrm{M}_{1}, \mathbb{M}_{2}$ and $\mathrm{M}_{3}$ be three arbitrary CBBAs: P1.1 Nonnegativity: $\mathbb{C}\left(\mathrm{M}_{1}, \mathrm{M}_{2}\right) \geq 0$.

P1.2 Nondegeneracy: $\mathbb{C}\left(\mathbb{M}_{1}, \mathbb{M}_{2}\right)=1$ if and only if $\mathbb{M}_{1}=$ $\mathrm{M}_{2}$.

P1.3 Symmetry: $\mathbb{C}\left(\mathrm{M}_{1}, \mathrm{M}_{2}\right)=\mathbb{C}\left(\mathrm{M}_{2}, \mathrm{M}_{1}\right)$.

P1.4 Boundedness: $0 \leq \mathbb{C}\left(\mathrm{M}_{1}, \mathrm{M}_{2}\right) \leq 1$.
Definition 3 (The complex conflict coefficient between $C B$ $B A s$ )

The complex conflict coefficient between CBBAs $\mathrm{M}_{1}$ and $\mathrm{M}_{2}$, denoted as $\mathbb{K}_{C B B A}\left(\mathrm{M}_{1}, \mathbb{M}_{2}\right)$ is defined by

$$
\begin{aligned}
\mathbb{K}_{C B B A}\left(\mathbb{M}_{1}, \mathbb{M}_{2}\right) & =1-\mathbb{C}\left(\mathbb{M}_{1}, \mathbb{M}_{2}\right) \\
& =1-\frac{\sqrt{\left\langle\overrightarrow{\mathbb{M}}_{1}, \overrightarrow{\mathbb{M}}_{2}\right\rangle\left\langle\overrightarrow{\mathbb{M}}_{2}, \overrightarrow{\mathbb{M}}_{1}\right\rangle}}{\left\|\overrightarrow{\mathbb{M}}_{1}\right\|\left\|\overrightarrow{\mathbb{M}}_{2}\right\|} .
\end{aligned}
$$

Theorem 2 The $\mathbb{K}_{C B B A}$ has the properties for conflict measurement [2], including the non-negativity, symmetry, boundedness, extreme consistency, and insensitivity to refinement.

Property 2 Let $\mathbb{M}_{1}$ and $\mathrm{M}_{2}$ be arbitrary two CBBAs:

P2.1 Non-negativity: $\mathbb{K}_{C B B A}\left(\mathbb{M}_{1}, \mathbb{M}_{2}\right) \geq 0$.

P2.2 Symmetry: $\mathbb{K}_{C B B A}\left(\mathbb{M}_{1}, \mathbb{M}_{2}\right)=\mathbb{K}_{C B B A}\left(\mathbb{M}_{2}, \mathbb{M}_{1}\right)$.

P2.3 Boundedness: $0 \leq \mathbb{K}_{C B B A}\left(\mathbb{M}_{1}, \mathbb{M}_{2}\right) \leq 1$.

P2.4 Extreme consistency: 1) $\mathbb{K}_{C B B A}\left(\mathbb{M}_{1}, \mathbb{M}_{2}\right)=0$ iff for the focal elements $A_{i}$ and $A_{j}$ of $\mathrm{M}_{1}$ and $\mathrm{M}_{2}$, respectively, $\left.\left(\cup A_{i}\right) \cap\left(\cup A_{j}\right)=\emptyset ; 2\right) \mathbb{K}_{C B B A}\left(\mathbb{M}_{1}, \mathbb{M}_{2}\right)=1$ iff $\mathbb{M}_{1}$ is completely equal to $\mathrm{M}_{2}$.

P2.5 Insensitivity to refinement: for $\mathrm{M}_{1}$ and $\mathrm{M}_{2}$ refined from FOD $\Omega$ to $\Omega^{\prime}, \quad \mathbb{K}_{C B B A}\left(\mathbb{M}_{1}^{\Omega}, \mathbb{M}_{2}^{\Omega}\right)=$ $\mathbb{K}_{C B B A}\left(\mathbb{M}_{1}^{\Omega^{\prime}}, \mathbb{M}_{2}^{\Omega^{\prime}}\right)$.

The complex conflict coefficient is a generalization of Jiang's conflict coefficient [2].

\section{NUMERICAL EXAMPLE}

A numerical example is provided to illustrate the proposed method.

Example 1 Assume there are two CBBAs $\mathbb{M}_{1}$ and $\mathrm{M}_{2}$ in FOD $\Omega$ :

$$
\begin{array}{ll}
\mathbb{M}_{1}: & \mathbb{M}_{1}\left(\left\{A_{1}\right\}\right)=\alpha+\beta i, \mathbb{M}_{1}(\omega)=1-\alpha-\beta i \\
\mathbb{M}_{2}: & \mathbb{M}_{2}\left(\left\{A_{1}\right\}\right)=1-\alpha+\beta i, \mathbb{M}_{2}(\omega)=\alpha-\beta i .
\end{array}
$$

In Example $1, \mathbb{M}_{1}$ and $\mathbb{M}_{2}$ change according to $\alpha$ and $\beta$ as well as the subset of $\omega$. Here, $\alpha$ and $\beta$ are set within $[0,1]$. The subset $\omega$ is set as $\left\{\mathcal{A}_{2}\right\}$ and $\left\{\mathcal{A}_{1}, \mathcal{A}_{2}\right\}$, respectively.

When $\beta=0$, it means that $\mathbb{M}_{1}$ and $\mathbb{M}_{2}$ degrade into real numbers from complex numbers, so that $\mathrm{M}_{1}\left(\left\{\mathcal{A}_{1}\right\}\right)=$ 
$\mathbb{M}_{2}(\omega)=\alpha$ and $\mathbb{M}_{1}(\omega)=\mathbb{M}_{2}\left(\left\{\mathcal{A}_{1}\right\}\right)=1-\alpha$. Under this situation, no matter how the $\omega$ changes as $\left\{A_{1}\right\}$ or $\left\{\mathcal{A}_{1}, \mathcal{A}_{2}\right\}$, we notice that the proposed $\mathbb{C}\left(\mathbb{M}_{1}, \mathbb{M}_{2}\right)$ is exactly the same as the Jiang's conflict coefficient method as shown in Fig. 1(a) and Fig. 1(b). This result verifies that when the CBBAs are degraded from complex numbers into real numbers, the CECC degrades into Jiang's conflict coefficient.

When $\alpha=0$, and $\omega=\left\{\mathcal{A}_{2}\right\}$, or $\phi=1$, and $\omega=\left\{\mathcal{A}_{2}\right\}$, the proposed correlation coefficient and Jiang's method have the minimal value of 0 . This result is reasonable, since under these cases there are no intersection between $\mathbb{M}_{1}$ and $\mathbb{M}_{2}$. Whereas, when $\phi=0$, and $\omega=\left\{\mathcal{A}_{1}, \mathcal{A}_{2}\right\}$, the proposed correlation coefficient and Jiang's method have the minimal value of 0.5 . This result is also reasonable, since under these cases there is an intersection $\left\{\mathcal{A}_{1}\right\}$ between $\mathbb{M}_{1}$ and $\mathbb{M}_{2}$. Therefore, the correlation coefficient measures are greater than zero.

Moreover, from the results shown in Fig. 1(a) and Fig. 1(b), it is obvious that the the properties for conflict measurement are verified, including the non-negativity, symmetry, boundedness, extreme consistency, and insensitivity to refinement.

\section{CONCLusions}

In this paper, a complex evidential correlation coefficient, called CECC, was proposed for complex mass functions in a more general framework of complex plane space. In addition, the properties of the proposed CECC were defined and analyze, where it has the properties of nonnegativity, nondegeneracy, symmetry and boundedness. On the basis of the CECC, a complex conflict coefficient was proposed for complex mass functions. A numerical example is provided to illustrate the proposed method.

The main contribution in this paper is that it is the first work to consider the conflict measure between pieces of evidence in the framework of a complex plane.

\section{CONFLICT OF INTEREST}

The author states that there are no conflicts of interest.

\section{ACKNOWLEDGMENTS}

This research is supported by the Fundamental Research Funds for the Central Universities (No. XDJK2019C085) and Chongqing Overseas Scholars Innovation Program (No. cx2018077).

\section{REFERENCES}

[1] F. Xiao, "Generalization of Dempster-Shafer theory: A complex mass function," Applied Intelligence, pp. DOI: 10.1007/s10 489-019-01 617-y, 2019.

[2] W. Jiang, "A correlation coefficient for belief functions," International Journal of Approximate Reasoning, vol. 103, pp. 94-106, 2018. 